\title{
Overall processes capability index for assembly production lines
}

\begin{abstract}
This study develops a new method called Overall Processes Capability Index (OPCI) to measure the capability of assembly lines. After defining relation between percentage yield and process capability, the new definition of overall defective rate is developed for assembly lines in different cases (series and parallel processes). The interesting point is that a rejected item from one process can be assembled with one another rejected item of another process and creates one accepted assembled one. Then regarding to this point and with the aid of transforming new definition of overall defective rate to process yield, the overall processes capability of assembly production line is measured.
\end{abstract}

Keyword: Process capability index; Defective rate; Process yield; Overall processes capability; Assembly lines. 